\title{
La Pedagogía de la muerte en el contexto de la pandemia: Una mirada radical e inclusiva
}

\author{
The Pedagogy of Death in the Context of the Pandemic: A Radical and Inclusive View
}

\section{A Pedagogia da morte no contexto da pandemia: Um olhar radical e inclusivo}

Agustín de la Herrán-Gascón

Universidad Autónoma de Madrid

Madrid, España

agustin.delaherran@uam.es

iD https://orcid.org/0000-0001-9156-6971

Resumen: En el actual escenario de pandemia, se ha intensificado el interés por la Pedagogía de la muerte. Es una disciplina científica cuyo objeto de estudio es la educación que incluye la muerte, como parte de una educación de la conciencia.

Palabras claves: Pedagogía de la muerte; Pedagogía radical e inclusiva; educación; conciencia; COVID-19.

Abstract: In the current pandemic scenario, interest in the Pedagogy of Death has intensified. It is a scientific discipline whose object of study is education that includes death, as part of an education of conscience.

Keywords: Pedagogy of death; radical and inclusive pedagogy; education; conscience; COVID-19.

Resumo: No actual cenário pandémico, o interesse pela Pedagogia da Morte intensificou-se. É uma disciplina científica cujo objecto de estudo é a educação que inclui a morte, como parte de uma educação de consciência.

Palavras-chaves: Pedagogia da norte; pedagogia radical e inclusiva; educação; consciencia; COVID-19.

Recibido: 02/08/2020 Aceptado: 10/08/2020 
https://doi.org/10.15359/ree.24-S.4

https://www.revistas.una.ac.cr/index.php/educare

educare@una.ac.cr

\section{Introducción}

La pandemia vigente fue declarada oficialmente por la OMS el 11 de marzo de 2020. La experiencia para la humanidad está siendo traumática, porque, si aquel día se registraban 4291 fallecidos en 114 países, hoy 3 de agosto de 2020, son 690.000 en casi todo el mundo. La situación ha intensificado el interés por la articulación entre la muerte y la educación y, por ende, por la Pedagogía de la muerte. El objetivo de este escrito es ponderar críticamente el interés y alcance hacia la educación que incluye la muerte y a la Pedagogía de la muerte, en el actual escenario de pandemia.

\section{Desarrollo}

No es edificante verificar que la educación no se entiende como locomotora social, sino como un vagón de cola esencialmente adaptativo, cuyos fines están supeditados a otras metas no pedagógicas. Quizá por una baja cultura general generalizada, la educación no se comprende y se utiliza, y se utiliza porque no se comprende. Por lo mismo, tampoco es de dominio general el conocimiento de la ciencia que la tiene como objeto de estudio: la Pedagogía.

Pero la Pedagogía tiene vacíos epistemológicos, sin cuya inclusión educativa y curricular difícilmente estudiará cabalmente la educación. Algunas de sus carencias, como ocurre con las raíces de un árbol, son esenciales, pero no están a la vista, son radicales. A su estudio y vinculación con la educación ordinaria se dedica la reciente Pedagogía radical e inclusiva. Los temas radicales, a diferencia de los disciplinares y transversales, incluidas las competencias, son fundamentales para la educación, pero no se demandan socialmente.

Uno de estos retos radicales es la conciencia de muerte y de finitud. Desde la perspectiva radical e inclusiva, si la educación se discerniese con mayor complejidad de conciencia, no se educaría para la vida, tal y como habitualmente se hace, por dos razones. Porque, normalmente se excluye la muerte, y, si no se incluye la muerte en esa vida, ¿sobre qué estaremos educando? Y porque la vida humana, tanto social como personalmente, es globalmente un desastre, parcialmente relacionado con la educación que se prodiga. Por tanto, tendría más sentido educar para cambiar la vida y hacerlo, además, radicalmente.

Para hacerlo, es imprescindible considerar la educación que incluye la muerte, junto a otros temas radicales, prácticamente inéditos: el ego humano, la conciencia, el autoconocimiento, la meditación, el amor, la humanidad, etc. Ante el reto de global de formación profunda, la muerte siempre ha ido por delante. Es algo que saben bien las escuelas espirituales y filosóficas de Oriente y Occidente. Desde la mirada de la Pedagogía radical e inclusiva, el estudio de la educación que tiene en cuenta la muerte no viene sola, porque a través suya conecta con un campo mayor, la educación basada en la conciencia. 
https://doi.org/10.15359/ree.24-S.4

Si la Pedagogía de la muerte es la disciplina científica que, desde hace treinta años estudia la educación que tiene en cuenta la muerte, la educación de la conciencia de muerte y de finitud define un atrio que comunica la educación ordinaria y la educación de la conciencia. Tanto la educación que incluye la muerte como la educación basada en la conciencia son campos emergentes relacionados entre sí, como la raíz con su árbol. ¿Queremos los frutos? Sí, pero si de entrada colocamos nuestra atención en los efectos, estaremos razonando al revés, sin apercibirnos de ello.

Con esta caución, relativa a la naturaleza epistemológica de la Pedagogía de la muerte, nos cuestionamos: ¿cuáles son algunos de sus objetos de estudio? Proponemos algunos: pseudo educación y educación radical e inclusiva; la conciencia de muerte y de finitud en la sociedad actual: del tabú al imperativo educativo; la Pedagogía radical e inclusiva y la pseudo educación; la muerte en los sistemas educativos actuales y en sus currículos; los múltiples conceptos de muerte aplicables a la educación: más allá del sufrimiento y del duelo; los tutores y los padres como protagonistas de la Pedagogía de la muerte; enfoques de la Pedagogía y didáctica de la muerte: metodología didáctica, recursos, evaluación, acompañamiento educativo, etc.; la Pedagogía de la muerte en los proyectos educativos institucionales: la planificación del periodo de duelo; formación de educadores en Pedagogía de la muerte; la educación que incluye la muerte y la educación radical o basada en la conciencia; innovación e investigación en Pedagogía de la muerte, etc. (de la Herrán Gascón et al., 2000; de la Herrán Gascón y Cortina Selva, 2006; Herrán Gascón y Rodríguez Herrero, 2017).

\section{Conclusiones}

Pocas veces la muerte ha golpeado tanto en tantas escuelas de todo el mundo. El principal fin de la educación, no contemplado aún por la Pedagogía, es el despertar de la conciencia humana, más allá del ego. La pandemia puede tener el valor de ayudar a despertar, un poco, a esta realidad científica y profesional emergente. Ahora bien, habría dos formas bienintencionadas de hacerlo. La primera sería la ordinaria, impulsiva o egocéntrica. Consistiría en actuar creyendo que el alumno es el centro de la educación, lo que comportaría tomar la acción o la práctica como referentes y desarrollarla. La segunda sería la consciente y radical. Entendería que el primer centro de la educación que hay que atender es el ego y la conciencia de uno mismo, sobre todo si de educadores se trata.

Nota general: Corrección de estilo y traducciones a cargo del autor. 
https://doi.org/10.15359/ree.24-S.4

https://www.revistas.una.ac.cr/index.php/educare

educare@una.ac.cr

\section{Referencias}

De la Herrán Gascón, A., González, I., Navarro, M. J., Bravo, S. y Freire, M. V. (2000). ¿Todos los caracoles se mueren siempre? Como tratar la muerte en educación infantil. De la Torre.

De la Herrán Gascón, A. y Cortina Selva, M. (2006). La muertey su didáctica. Manual para educación infantil, primaria y secundaria (2. ${ }^{a}$ ed.). Universitas.

De la Herrán Gascón, A. y Rodríguez Herrero, P. (Dirs.) (2017). Innovación e inclusión de la muerte en la educación. Proyectos de I+D+i, del Programa Estatal de Investigación, Desarrollo e Innovación Orientada a los Retos de la Sociedad. Ministerio de Ciencia, Innovación y Universidades de España y Fondo Europeo de Desarrollo Regional (FEDER). Ref.: EDU201785296-R (AEI/FEDER, UE). Recuperado de https://pedagogiadelamuerte.com/ 